Georgian Mathematical Journal

Volume 12 (2005), Number 2, 349-368

\title{
ON THE STRONG DIFFERENTIATION OF MULTIPLE INTEGRALS ALONG DIFFERENT FRAMES
}

\author{
GIORGI ONIANI
}

\begin{abstract}
Let $E$ be a set consisting of rectangular frames in $\mathbb{R}^{n}$. The following question connected with one problem of A. Zygmund is studied in the paper: Does there exists a function the integral of which is: 1) nondifferentiable almost everywhere in a strong sense along every frame from $E$, and 2) strongly differentiable along every frame not belonging to $E$ ? In particular, the question is solved on the existence of a non-empty set $E$ different from the set of all rectangular frames for which there is a function with the properties 1) and 2).
\end{abstract}

2000 Mathematics Subject Classification: 28A15.

Key words and phrases: Strong differentiation, Lebesgue multiple integral, differentiation basis, frame.

\section{Definitions and the Notation}

A mapping $B$ defined on $\mathbb{R}^{n}$ is said to be a differentiation basis in $\mathbb{R}^{n}$ if for every $x \in \mathbb{R}^{n}, B(x)$ is a family of open bounded sets containing the point $x$ such that there exists a sequence $\left\{R_{k}\right\} \subset B(x)$ with diam $R_{k} \rightarrow 0(k \rightarrow \infty)$.

Let $B$ be a differentiation basis in $\mathbb{R}^{n}$. For $f \in L_{l o c}\left(\mathbb{R}^{n}\right)$ and $x \in \mathbb{R}^{n}$, the upper and lower limits of the integral mean $\frac{1}{|R|} \int_{R} f$, where $R$ is an arbitrary set from $B(x)$ and diam $R \rightarrow 0$, are called the upper and the lower derivatives with respect to $B$ of the integral of $f$ at the point $x$, and are denoted by $\bar{D}_{B}\left(\int f, x\right)$ and $\underline{D}_{B}\left(\int f, x\right)$, respectively. When they are equal, their common value is called a derivative with respect to $B$ of $\int f$ at the point $x$ and is denoted by $D_{B}\left(\int f, x\right)$. The basis $B$ is said to differentiate the integral of $f$ if $D_{B}\left(\int f, x\right)=f(x)$ for almost all $x$. Under $M_{B}$ we mean the maximal operator corresponding to $B$, i.e., $M_{B}(f)(x)=\sup _{R \in B(x)} \frac{1}{|R|} \int_{R}|f|\left(f \in L_{l o c}\left(\mathbb{R}^{n}\right), \quad x \in \mathbb{R}^{n}\right)$. Also let $M_{B}^{(r)}(f)(x)=$ $\sup _{R \in B(x), \operatorname{diam} R<r} \frac{1}{|R|} \int_{R}|f|$ for any $r>0$.

In what follows the dimension of the considered space $\mathbb{R}^{n}$ is assumed to be greater than or equal to 2 .

By $\mathbb{I}\left(\mathbb{R}^{n}\right)(n \in \mathbb{N})$ we denote the differentiation basis in $\mathbb{R}^{n}$ for which $\mathbb{I}\left(\mathbb{R}^{n}\right)(x)$ $\left(x \in \mathbb{R}^{n}\right)$ consists of all $n$-dimensional intervals containing $x$. When the value of the considered dimension is known, we simply write $\mathbb{I}$.

A set whose elements are $n$ mutually orthogonal straight lines passing through the origin 0 is called a frame in the space $\mathbb{R}^{n}$. Denote such a frame by $\theta$ $\left(\theta=\left\{\theta_{1}, \ldots, \theta_{n}\right\}\right)$. Under $\theta_{0}$ we understand the frame $\left\{0 x_{1}, \ldots, 0 x_{n}\right\}$, where 
$0 x_{1}, \ldots, 0 x_{n}$ are the coordinate axes of $\mathbb{R}^{n}$. Denote the set of all frames by $\theta\left(\mathbb{R}^{n}\right)$.

We call $\theta=\left\{\theta_{1}, \ldots, \theta_{n}\right\}$ the frame of a rectangular parallelepiped $I$ in $\mathbb{R}^{n}$ and denote it by $\theta(I)$ if the edges of $I$ are parallel to the corresponding lines $\theta_{i}$ $(i \in \overline{1, n})$.

For any given nonempty set $E \subset \theta\left(\mathbb{R}^{n}\right)$ we denote by $\mathbb{I}(E)$ the differentiation basis for which $\mathbb{I}(E)(x)\left(x \in \mathbb{R}^{n}\right)$ consists of all rectangular parallelepipeds $I$ with the properties: $x \in I, \theta(I) \in E$. If $E=\{\theta\}$, instead of $\mathbb{I}(\{\theta\})$ we simply write $\mathbb{I}(\theta)$. It is clear that $\mathbb{I}\left(\theta_{0}\right)=\mathbb{I}$.

Differentiability of an integral with respect to $\mathbb{I}(\theta)$ is sometimes called strong differentiability along the frame $\theta$. In the case $\theta=\theta_{0}$ it is called strong differentiability.

Let $1 \leq k \leq n-1$. We call a set $E \subset \theta\left(\mathbb{R}^{n}\right)$ an orbit of $k$ th order if there exist a set $\Delta=\Delta_{E}$ consisting of $n-k$ mutually orthogonal and passing through the origin lines such that $E=\left\{\theta \in \theta\left(\mathbb{R}^{n}\right): \Delta \subset \theta\right\}$. It is natural to consider $\varnothing$ and $\theta\left(\mathbb{R}^{n}\right)$ as orbits of 0 th and $n$th order, respectively.

We call a set $E \subset \theta\left(\mathbb{R}^{n}\right)$ an orbit if $E$ is an orbit of $k$ th order for some $k \in \overline{0, n}$. By $k_{E}$ we denote the order of an orbit $E$. We call an orbit $E$ nontrivial if $0<k_{E}<n$.

Let $G^{n}=(0,1)^{n}$. Let us agree to denote by $\Phi(L)\left(G^{n}\right)$ the class of all measurable functions $f: \mathbb{R}^{n} \rightarrow \mathbb{R}$ having the following properties: $\operatorname{supp} f \subset G^{n}$, $\int_{G^{n}} \Phi(|f|)<\infty$

\section{Statement of the Problem and the Result}

According to the classical theorem of Jessen, Marcinkiewicz and Zygmund [1], if $f \in L\left(\ln ^{+} L\right)^{n-1}\left(G^{n}\right)$, then $\int f$ is strongly differentiable. This result does not extend to arbitrary integrable functions; moreover, in any class $\Phi(L)\left(G^{n}\right)$ wider than $L\left(\ln ^{+} L\right)^{n-1}\left(G^{n}\right)$, Saks [2] constructed the function whose integral is nondifferentiable a.e. in a strong sense on $G^{n}$. Since Saks' construction was tied to the particular orientation of the axes, Zygmund [3, p. 99] posed the following problem:

Given $f \in L\left(\mathbb{R}^{2}\right)$, is it possible to choose a pair of rectangular directions so that if $B$ is the basis of all open rectangles with sides in those directions, then $B$ differentiates $\int f$ ? If the answer is affirmative, what is the set of all eligible directions with this property?

Marstrand [4] showed that the choice of a "good" pair of rectangular directions (i.e., a frame) cannot be made for an arbitrary function, namely, he constructed the function whose integral, for any frame $\theta$, is strongly nondifferentiable along $\theta$ a.e. on $G^{2}$. In connection with the problem of Zygmund and its negative solution there naturally arises question: Does there exists a function the integral of which is not strongly differentiable, but there is a frame $\theta$ along which $\int f$ is strongly differentiable? In general, of what kind can be sets of "differentiability frames" and "non-differentiability frames" for a given function? 
Let us set $E \subset \theta\left(\mathbb{R}^{n}\right)$ call an $R$-set if there exists a function $f \in L\left(G^{n}\right)$ such that for every $\theta \in E$ we have $\bar{D}_{\mathbb{I}(\theta)}\left(\int f, x\right)=\infty$ a.e. on $G^{n}$ and for every $\theta \notin E$ the integral $\int f$ is strongly differentiable along $\theta$.

Now the problem can be formulated as follows: what kind of sets $E \subset \theta\left(\mathbb{R}^{n}\right)$ are $R$-sets?

Obviously, $\varnothing$ is an $R$-set. On the other hand, due to the results of Marstrand [4] and Lopez-Melero [5] $\theta\left(\mathbb{R}^{n}\right)$ is an $R$-set too.

In studying the above problem, a question arises whether there in general exists an $R$-set different from $\varnothing$ and $\theta\left(\mathbb{R}^{n}\right)$ ?

A positive answer to this question for the two-dimensional case follows from the results of Lepsveridze [6], Oniani [7] and Stokolos [8], while for the general case it is given here in the form of the following theorem.

Theorem. Every union of a finite number of orbits is an R-set.Moreover, if $E \subset \theta\left(\mathbb{R}^{n}\right)$ is a union of a finite number of orbits, then for every function $f \in L \backslash L\left(\ln ^{+} L\right)^{n-1}\left(G^{n}\right), f \geq 0$, there exists an equimeasurable with $f$ function $g: \mathbb{R}^{n} \rightarrow \mathbb{R}$, supp $g \subset G^{n}$, such that:

1) for every $\theta \in E \quad \bar{D}_{\mathbb{I}(\theta)}\left(\int g, x\right)=\infty$ a.e. on $G^{n}$;

2) for every $\theta \notin E \quad \int g$ is strongly differentiable along $\theta$.

Remark 1. This theorem was announced in [9] for a finite union of orbits of $(n-1)$ th order, and in [10] for the general case. In [11] that was published between [9] and [10] it was announced that for any $n \geq 2$ every finite set is an $R$-set.

Remark 2. Note that our theorem generalizes the corresponding results of Saks [2], Marstrand [4] and Stokolos [12].

Remark 3. In the two-dimensional case, a more complete result than the above theorem is valid. In particular, in [7] (see also [13]) we prove: no more than a countable set $E \subset \theta\left(\mathbb{R}^{2}\right)$ is an $R$-set if and only if $E$ is of type $G_{\delta}$ with respect to the natural metric in $\theta\left(\mathbb{R}^{2}\right)$. Mention should be made of [14], where some generalizations of the results from [7] are given.

\section{Some Hints for the Proof of the Theorem}

For $E=\theta\left(\mathbb{R}^{n}\right)$, all main ideas of the proof are available from $[4,5]$. The proof is almost trivial for $E=\varnothing$. Hence we will dwell on the case where $E$ is the union of a finite number of nontrivial orbits. In this case, the proof is based on the following assertion: for a given nontrivial orbit $E$ and any numbers $\varepsilon>0$, $h>1$ there exists a rectangular parallelepiped $I$ for which a measure of the set $\left\{M_{\mathbb{I}(\theta)}\left(h \chi_{I}\right)>1\right\}$ is "large" (of order $\left.h(\ln h)^{n-1}|I|\right)$ when $\theta \in E$, and is "small" (of order not larger than $h(\ln h)^{n-2}|I|$ ) when $\theta \in E_{\varepsilon}$, where, as $\varepsilon$ decreases, $E_{\varepsilon}$ exhausts the set $\theta\left(\mathbb{R}^{n}\right) \backslash E$. Such a contrast between $\theta \in E$ and $\theta \in E_{\varepsilon}$ takes place on a rectangular parallelepiped $I$ with the edges $\delta_{1}, \ldots, \delta_{n}$ such that $\delta_{1}<\cdots<\delta_{n-k_{E}-1}=\cdots=\delta_{n}$ and $\frac{\delta_{i+1}}{\delta_{i}}$ is "large enough" for every $i \in \overline{1, n-k_{E}}$. We also establish that the the sets $\left\{M_{\mathbb{I}(\theta)}\left(h \chi_{I}\right)>1\right\}$ can be approximated (from below when $\theta \in E$, and from above when $\theta \in E_{\varepsilon}$ ) by sets which can in turn be 
"uniformly approximated" by finite unions of diadic cubes of fixed order. This fact allows us to apply Marstrand's method of accumulation of singularities. Both above assertions are contained in the assertion which we call the Lemma on Contrast.

Note that to have the convergence effect we make an essential use of the assertion which we call the Lemma on Separation.

\section{BASIC Lemmas}

Let $E$ be a nontrivial orbit. By $\sigma(E)$ denote the collection of all bijections from $\overline{0, n-k_{E}}$ into $\Delta_{E}$, and for $\sigma \in \sigma(E), 0<\varepsilon<\pi / 4$, introduce the notation:

I. Let $2 \leq j \leq n$. By $E_{1, j}(\sigma, \varepsilon)$ denote the set of all frames $\theta \in \theta\left(\mathbb{R}^{n}\right)$ for which, among straight lines from $\theta, j$ form, with $\sigma(1)$, angles less than $\pi / 2-\varepsilon$, while the remaining lines from $\theta$ are orthogonal to $\sigma(1)$.

II. Let $2 \leq i \leq n-k_{E}$ and $2 \leq j \leq n-i+1$. By $E_{i, j}(\sigma, \varepsilon)$ denote the set of all frames $\theta \in \theta\left(\mathbb{R}^{n}\right)$ for which:

1) $\sigma(1), \ldots, \sigma(i-1) \in \theta$,

2) among lines from $\theta \backslash\{\sigma(1), \ldots, \sigma(i-1)\}, j$ ones form, with $\sigma(i)$, angles less than $\pi / 2-\varepsilon$, while the remaining lines from $\theta \backslash\{\sigma(1), \ldots, \sigma(i-1)\}$ are orthogonal to $\sigma(i)$.

III. $\quad E(\sigma, \varepsilon)=\bigcup_{i=1}^{n-k_{E}} \bigcup_{j=1}^{n-j+1} E_{i, j}(\sigma, \varepsilon)$.

Note that for any orbit $E$ and $\sigma \in \sigma(E)$

$$
E(\sigma, \varepsilon) \subset E(\sigma, \delta) \text { if } \delta<\varepsilon, \text { and } \bigcup_{0<\varepsilon<\pi / 4} E(\sigma, \varepsilon)=\theta\left(\mathbb{R}^{n}\right) \backslash E \text {. }
$$

Recall that a rectangular parallelepiped in $\mathbb{R}^{n}$ is a set of the form

$$
\left\{x \in \mathbb{R}^{n}:\left|\left(e_{1}, x-x_{0}\right)\right|<\delta_{1}, \ldots,\left|\left(e_{n}, x-x_{0}\right)\right|<\delta_{n}\right\},
$$

where $\left\{e_{1}, \ldots, e_{n}\right\}$ is an orthonormal basis in $\mathbb{R}^{n}, x_{0} \in \mathbb{R}^{n}$, and $\delta_{1}, \ldots, \delta_{n}>0$, and its edges are segments of the form

$$
\left\{x \in \mathbb{R}^{n}:\left|\left(e_{i}, x-x_{0}\right)\right|<\delta_{i}, \quad\left(e_{j}, x-x_{0}\right)=\varepsilon_{j} \delta_{j}(j \in \overline{1, n}, \quad j \neq i)\right\},
$$

where $i \in \overline{1, n}$ and $\left|\varepsilon_{j}\right|=1(j \in \overline{1, n}, j \neq i)$. As is easily seen, the edges corresponding to fixed $i \in \overline{1, n}$ are parallel to the vector $e_{i}$ and have a length equal to $2 \delta_{i}$.

In what follows, for simplicity, we write "rectangle" instead of "rectangular parallelepiped" and "interval" instead of " $n$-dimensional interval".

Let $E$ be a nontrivial orbit. For $\sigma \in \sigma(E)$, denote by $\mathcal{I}(E, \sigma)$ the collection of all rectangles $I$ such that

$$
I=\left\{x \in \mathbb{R}^{n}:\left|\left(e_{1}, x-x_{0}\right)\right|<\delta_{1}, \ldots,\left|\left(e_{n}, x-x_{0}\right)\right|<\delta_{n}\right\},
$$

where $\delta_{1}<\cdots<\delta_{n-k_{E}+1}=\cdots=\delta_{n}$ and $e_{1}, \ldots, e_{n-k_{E}}$ are parallel to the lines $\sigma(1), \ldots, \sigma\left(n-k_{E}\right)$, respectively. Denote also $\mathcal{I}(E)=\bigcup_{\sigma \in \sigma(E)} \mathcal{I}(E, \sigma)$.

For $E=\theta\left(\mathbb{R}^{n}\right)$, by $\mathcal{I}(E)$ denote the collection of all cubes in $\mathbb{R}^{n}$. 
Let $E$ be a nontrivial orbit. For $I \in \mathcal{I}(E)$ having pairwise orthogonal edges of length $\delta_{1}, \ldots, \delta_{n}$, where $\delta_{1}<\cdots<\delta_{n-k_{E}+1}=\cdots=\delta_{n}$, denote

$$
r(I)=\min \left\{\frac{\delta_{i+1}}{\delta_{i}}: i \in \overline{1, n-k_{E}}\right\} .
$$

In what follows $\chi_{A}$ always stands for the characteristic function of a set $A \subset \mathbb{R}^{n}$.

For a set $E \subset \mathbb{R}^{n}$ having the center of symmetry and for $h>0$, by $h E$ denote the image of $E$ under the homothety with the coefficient $h$ and center coinciding with the center of $E$.

In the inequalities given below, by $c_{1}(n), c_{2}(n), \ldots$ we denote the positive constants depending only on the parameter $n$.

Lemma on Contrast. The following two assertions are valid:

I. Let $E$ be a nonempty orbit and $h>1$. If a rectangle $I$ belongs to $\mathcal{I}(E)$, then there exists a collection $\{T(\theta): \theta \in E\}$ such that:

1) for every $\theta \in E, T(\theta)$ is a union of a finite number of balls of fixed radius; moreover, the radius and the number of balls are the same for every $\theta \in E$;

2) for every $\theta \in E$,

$$
\begin{aligned}
& \left\{M_{\mathbb{I}(\theta)}\left(h \chi_{I}\right)>1\right\} \supset T(\theta), \\
& T(\theta) \subset 2^{2 n} h I, \\
& |T(\theta)| \geq c_{1}(n) h(\ln h)^{n-1}|I| ;
\end{aligned}
$$

II. Let $E$ be a nontrivial orbit, $h>1, \sigma \in \sigma(E)$ and $0<\varepsilon<\pi / 4$. If I belongs to $\mathcal{I}(E, \sigma)$ and $r(I)>\frac{4 n \sqrt{n} h}{\sin \varepsilon}$, then there exists a collection $\{T(\theta): \theta \in E(\sigma, \varepsilon)\}$ such that

1) for every $\theta \in E(\sigma, \varepsilon), T(\theta)$ is a union of a finite number of balls of fixed radius; moreover, the radius and the number of balls are the same for every $\theta \in E(\sigma, \varepsilon)$;

2) for every $\theta \in E(\sigma, \varepsilon)$

$$
\begin{aligned}
& \left\{M_{\mathbb{I}(\theta)}\left(h \chi_{I}\right)>1\right\} \subset T(\theta), \\
& T(\theta) \subset 2^{4 n+3} h I, \\
& |T(\theta)| \leq c_{2}(n) h(\ln h)^{n-2}|I| .
\end{aligned}
$$

It is obvious that in the case of a nontrivial orbit $E$ assertions I and II for $I \in \mathcal{I}(E, \sigma)$ give the above-mentioned contrast.

Denote by $\bar{L}\left(\mathbb{R}^{n}\right)$ the class of all functions $f \in L\left(\mathbb{R}^{n}\right)$ for everyone of which there exists, for any $\varepsilon>0$, a continuous function $g \in L\left(\mathbb{R}^{n}\right)$ on $\mathbb{R}^{n}$ such that $|g(x)| \leq|f(x)|$ a. e. on $\mathbb{R}^{n}$, and $\int_{\mathbb{R}^{n}}|f-g|<\varepsilon$.

In [15] the following lemma is proved (see also [13, p. 52]).

Lemma on Separation. Let $f_{k} \in \bar{L}\left(\mathbb{R}^{n}\right), f_{k} \geq 0(k \in \mathbb{N}), E \subset \theta\left(\mathbb{R}^{n}\right)$, $E \neq \varnothing, \lambda>0$, and for every $k, m \in \mathbb{N}, k \neq m$, let the following conditions be fulfilled:

$$
\operatorname{supp} f_{k} \cap \operatorname{supp} f_{m}=\varnothing \text {, }
$$




$$
\begin{aligned}
& \operatorname{supp} f_{k} \cap\left\{M_{\mathbb{I}(E)}\left(f_{m}\right)>\lambda\right\}=\varnothing, \\
& \left\{M_{\mathbb{I}(E)}\left(f_{k}\right)>\lambda\right\} \cap\left\{M_{\mathbb{I}(E)}\left(f_{m}\right)>\lambda\right\}=\varnothing .
\end{aligned}
$$

Then

$$
\left\{M_{\mathbb{I}(E)}\left(\sum_{k=1}^{\infty} f_{k}\right)>\lambda\right\}=\bigcup_{k=1}^{\infty}\left\{M_{\mathbb{I}(E)}\left(f_{k}\right)>\lambda\right\} .
$$

The function $F: \mathbb{R} \rightarrow[0,1]$ is called a distribution if $F$ is decreasing, $\lim _{\lambda \rightarrow-\infty} F(\lambda)=1, \lim _{\lambda \rightarrow \infty} F(\lambda)=0$ and $F$ is right-continuous.

By $F_{f}$ we denote the distribution function of a measurable function $f: \mathbb{R}^{n} \rightarrow$ $\mathbb{R}$, i.e., $F_{f}(\lambda)=|\{f>\lambda\}|(\lambda \in \mathbb{R})$.

It is obvious that for any measurable $f: \mathbb{R}^{n} \rightarrow \mathbb{R}, F_{f}$ is a distribution.

Lemma on Distribution. Suppose that the distribution $F$ is such that $F(\lambda)=1$ for $\lambda<0$. Let $E \subset G^{n}$ and $|E| \geq F(0)$. Then there exists a function $f \in L\left(G^{n}\right)$ with the properties: $F_{f}=F$, supp $f \subset E, \int f$ is strongly differentiable along every frame.

\section{Some Auxiliary Propositions}

In this section we give some simple propositions that we need for proving of the Lemma on Contrast.

Taking into account that rotation is a measure preserving mapping, one can easily verify

Proposition 1. Let $f: \mathbb{R}^{n} \rightarrow \mathbb{R}$ be a measurable function. Then for every $\theta \in \theta\left(\mathbb{R}^{n}\right)$

$$
\begin{aligned}
& M_{\mathbb{I}(\theta)}(f)(x)=M_{\mathbb{I}}\left(f \circ \gamma_{\theta}\right)\left(\gamma_{\theta}^{-1}(x)\right) \quad\left(x \in \mathbb{R}^{n}\right), \\
& \left|\left\{M_{\mathbb{I}(\theta)}(f)>\lambda\right\}\right|=\left|\left\{M_{\mathbb{I}}\left(f \circ \gamma_{\theta}\right)>\lambda\right\}\right| \quad(\lambda>0),
\end{aligned}
$$

where $\gamma_{\theta}$ denotes rotation such that $\left\{\gamma_{\theta}\left(0 x_{1}\right), \ldots, \gamma_{\theta}\left(0 x_{n}\right)\right\}=\theta$.

By virtue of the obvious equality $\left\{M_{\mathbb{I}}(f)>\lambda\right\}=\left\{M_{\mathbb{I}}\left(f \chi_{\{|f|>\lambda / 2\}}\right)>\lambda / 2\right\}$, the well-known strong maximal inequality (see e.g. [3, p. 51]) and Proposition 1 give rise to

Proposition 2. Let $\lambda>0$ and

$$
\int_{\{|f|>\lambda / 2\}} \frac{|f|}{\lambda}\left(1+\ln ^{+} \frac{2|f|}{\lambda}\right)^{n-1}<\infty .
$$

Then for every $\theta \in \theta\left(\mathbb{R}^{n}\right)$

$$
\left|\left\{M_{\mathbb{I}(\theta)}(f)>\lambda\right\}\right| \leq c_{3}(n) \int_{\{|f|>\lambda / 2\}} \frac{|f|}{\lambda}\left(1+\ln ^{+} \frac{2|f|}{\lambda}\right)^{n-1} .
$$

An open set bounded by two different parallel hyperplanes, i.e., a set of the form $\left\{x \in \mathbb{R}^{n}:\left|\left(e, x-x_{0}\right)\right|<\delta\right\}$, where $e \in \mathbb{R}^{n}$ is the unit vector, $x_{0} \in \mathbb{R}^{n}$ and $\delta>0$, is called a strip in $\mathbb{R}^{n}$. The number $\delta$ is called the width of a strip. 
The basis $B$ in $\mathbb{R}^{n}$ is said to be convex if every $R \in B(x)$ is a convex set for every $x \in \mathbb{R}^{n}$.

In [15] the following assertion is proved (see also [13, p. 44]): Let $B$ be a convex basis in $\mathbb{R}^{n}$, $S$ be a strip in $\mathbb{R}^{n}$ of width $\delta$. Then $M_{B}\left(\chi_{S}\right)(x)<2^{n} \delta / \operatorname{dist}(x, S)$ when $\operatorname{dist}(x, S) \geq \delta$ (note that for $n=2$ and $B=\mathbb{I}\left(\mathbb{R}^{2}\right)$ the assertion was proved earlier in [7] and [8], a sharper estimate being obtained in [8]).

Majorizing the rectangle by strips generated by its parallel faces and using the above assertion in each case, one can easily prove

Proposition 3. Let $B$ be a convex basis in $\mathbb{R}^{n}, I$ be a rectangle in $\mathbb{R}^{n}$ and $h>1$. Then

$$
\left\{M_{B}\left(h \chi_{I}\right)>1\right\} \subset\left(2^{n} h+1\right) I .
$$

Therefore, if $f: \mathbb{R}^{n} \rightarrow \mathbb{R}$ is a measurable bounded function with bounded support, then for every $\lambda>0$ the set $\left\{M_{B}(f)>\lambda\right\}$ is bounded.

Let $B_{1}$ and $B_{2}$ be bases in $\mathbb{R}^{n_{1}}$ and $\mathbb{R}^{n_{2}}$, respectively. The product of $B_{1}$ and $B_{2}$ is denoted by $B_{1} \times B_{2}$ and defined as follows:

$$
B_{1} \times B_{2}\left(x_{1}, x_{2}\right)=\left\{R_{1} \times R_{2}: R_{1} \in B_{1}\left(x_{1}\right), \quad R_{2} \in B_{2}\left(x_{2}\right)\right\} .
$$

The basis $B$ in $\mathbb{R}^{n}$ is said to be translation invariant if for every $x \in \mathbb{R}^{n}$ $B(x)=\{x+R, R \in B(0)\}$, where 0 is the origin.

Based on Propositions 2 and 3 , by a standard technique it is easy to prove

Proposition 4. Let $B_{1}$ and $B_{2}$ be translation invariant convex bases in $\mathbb{R}^{n_{1}}$ and $\mathbb{R}^{n_{2}}$, respectively. For every continuous function $f: \mathbb{R}^{n_{1}+n_{2}} \rightarrow \mathbb{R}$ and $x_{1} \in \mathbb{R}^{n_{1}}, x_{2} \in \mathbb{R}^{n_{2}}$ assume that

$$
\begin{aligned}
& M_{1}(f)\left(x_{1}, x_{2}\right)=M_{B_{1}}\left[f\left(\cdot, x_{2}\right)\right]\left(x_{1}\right)=\sup _{R \in B\left(x_{1}\right)} \frac{1}{|R|_{n_{1}}} \int_{R}\left|f\left(t, x_{2}\right)\right| d t \\
& M_{2}(f)\left(x_{1}, x_{2}\right)=M_{B_{2}}\left[f\left(x_{1}, \cdot\right)\right]\left(x_{2}\right)=\sup _{R \in B\left(x_{2}\right)} \frac{1}{|R|_{n_{2}}} \int_{R}\left|f\left(x_{1}, \tau\right)\right| d \tau .
\end{aligned}
$$

Then:

1) if $f: \mathbb{R}^{n_{1}+n_{2}} \rightarrow \mathbb{R}$ is uniformly continuous, then $M_{1}(f)$ and $M_{2}(f)$ are uniformly continuous too;

2) for every uniformly continuous $f: \mathbb{R}^{n_{1}+n_{2}} \rightarrow \mathbb{R}$

$$
M_{B_{1} \times B_{2}}(f) \leq M_{1}\left[M_{2}(f)\right]
$$

3) if $B_{1}=\mathbb{I}\left(\mathbb{R}^{n_{1}}\right), f: \mathbb{R}^{n_{1}+n_{2}} \rightarrow \mathbb{R}$ is a uniformly continuous function and for given $\lambda>0$

$$
\int_{\{|f|>\lambda / 2\}} \frac{|f|}{\lambda}\left(1+\ln ^{+} \frac{2|f|}{\lambda}\right)^{n_{1}-1}<\infty
$$

then

$$
\left|\left\{M_{1}(f)>\lambda\right\}\right| \leq c_{3}\left(n_{1}\right) \int_{\{|f|>\lambda / 2\}} \frac{|f|}{\lambda}\left(1+\ln ^{+} \frac{2|f|}{\lambda}\right)^{n_{1}-1}
$$


4) if $f: \mathbb{R}^{n_{1}+n_{2}} \rightarrow \mathbb{R}$ is a continuous bounded function with bounded support, then for every $\lambda>0$ the sets $\left\{M_{1}(f)>\lambda\right\}$ and $\left\{M_{2}(f)>\lambda\right\}$ are bounded.

The following assertion is easy to verify.

Proposition 5. Let $B$ be a translation invariant basis in $\mathbb{R}^{n}$ and let $f: \mathbb{R}^{n} \rightarrow$ $\mathbb{R}$ be a uniformly continuous function. Then $M_{B}(f)$ is uniformly continuous.

Proposition 6. Let $\Phi:[0, \infty) \rightarrow[0, \infty)$ be an absolutely continuous strictly increasing function such that $\Phi(0)=0$ and $\lim _{t \rightarrow \infty} \Phi(t)=\infty$, and let $f: \mathbb{R}^{n} \rightarrow \mathbb{R}$ be a measurable function such that $\int_{\mathbb{R}^{n}} \Phi(|f|)<\infty$. Then

$$
\int_{\mathbb{R}^{n}} \Phi(|f|)=\int_{0}^{\infty} F_{|f|}(\lambda) \Phi^{\prime}(\lambda) d \lambda
$$

Proof. It is easy to verify that $\lim _{\lambda \rightarrow 0+} \Phi(\lambda) F_{|f|}(\lambda)=0$ and $\lim _{\lambda \rightarrow \infty} \Phi(\lambda) F_{|f|}(\lambda)=0$. Now taking into account the equalities

$$
\int_{\mathbb{R}^{n}} \Phi(|f|)=-\int_{0}^{\infty} \Phi(\lambda) d F_{|f|}(\lambda)=-\lim _{k \rightarrow \infty} \int_{1 / k}^{k} \Phi(\lambda) d F_{|f|}(\lambda)
$$

and performing integration by parts in the last integral, we conclude that the proposition is valid.

\section{Proofs of the Basic Lemmas}

In [15] the following assertion is proved (see also [13, p. 77]).

Lemma 1. Let $h>1,0<\varepsilon<\pi / 4$, I be a rectangle in $\mathbb{R}^{n}$ having pairwise orthogonal edges of length $\delta_{1}, \ldots, \delta_{n}$, where $\delta_{i}>\frac{n h}{\sin \varepsilon} \delta_{1}(i \in \overline{2, n})$, and $\ell$ be $a$ straight line passing through the origin and parallel to the edges of length $\delta_{1}$. Then for every frame $\theta \in \theta\left(\mathbb{R}^{n}\right)$ whose every line forms with $\ell$ angles less than $\pi / 2-\varepsilon$ we have

$$
\left|\left\{M_{\mathbb{I}(\theta)}\left(h \chi_{I}\right)>1\right\}\right| \leq 9^{n} h|I| .
$$

Lemma 2. Let $n \geq 3, h>1,0<\varepsilon<\pi / 4$, and let $A=E \times(-\delta, \delta)$, where $E \subset \mathbb{R}^{n-1}$ is an open bounded convex set containing a ball in $\mathbb{R}^{n-1}$ of radius not less than $\frac{n h}{\sin \varepsilon} \delta$. Then for every frame $\theta \in \theta\left(\mathbb{R}^{n}\right)$ whose every line forms with $0 x_{n}$ an angle less than $\pi / 2-\varepsilon$ we have

$$
\left|\left\{M_{\mathbb{I}(\theta)}\left(h \chi_{A}\right)>1\right\}\right| \leq n^{2 n} 9^{n} h|A| .
$$

Proof. According to John's lemma (see [3, p. 139]), there is an open ellipsoid $T$ such that $\frac{1}{n-1} T \subset E \subset T$. Let $R$ be a minimal rectangle containing $T$ and having edges parallel to the principal axes of $T$. It is easy to see that $\frac{1}{n-1} R \subset T \subset R$. Therefore $\frac{1}{(n-1)^{2}} R \subset E \subset R$. 
Since $E$ contains a ball of radius not less than $\frac{n h}{\sin \varepsilon} \delta$, every edge of $R$ is of length not less than $\frac{2 n h}{\sin \varepsilon} \delta$. If we set $I=R \times(-\delta, \delta)$, then from Lemma 1 we conclude: for $\theta=\left\{\theta_{1}, \ldots, \theta_{n}\right\}$ with $\angle\left(\theta_{i}, O x_{n}\right)<\pi / 2-\varepsilon(1 \leq i \leq n)$

$$
\begin{aligned}
\left|\left\{M_{\mathbb{I}(\theta)}\left(h \chi_{A}\right)>1\right\}\right| \leq\left|\left\{M_{\mathbb{I}(\theta)}\left(h \chi_{I}\right)>1\right\}\right| \\
\quad \leq 9^{n} h|I| \leq(n-1)^{2(n-1)} 9^{n} h|A|<n^{2 n} 9^{n} h|A| .
\end{aligned}
$$

The lemma is proved.

Lemma 3. Let $E$ be a nontrivial orbit, $0<\varepsilon<\pi / 4, \sigma \in \sigma(E), h>1$. If a rectangle $I$ belongs to $\mathcal{I}(E, \sigma)$ and $r(I)>\frac{2 n \sqrt{n} h}{\sin \varepsilon}$, then for every $\theta \in E(\sigma, \varepsilon)$

$$
\left|\left\{M_{\mathbb{I}(\theta)}\left(h \chi_{I}\right)>1\right\}\right| \leq c_{4}(n) h(\ln h)^{n-2}|I| .
$$

Proof. For $n=2$ the assertion follows from Lemma 1. Below we assume that $n \geq 3$.

First assume that $2 \leq i \leq n-k_{E}, 2 \leq j \leq n-i+1$, and $\theta \in E_{i, j}(\sigma, \varepsilon)$. Due to Proposition 1 it can be assumed without loss of generality that $\theta=$ $\theta_{0}=\left\{0 x_{1}, \ldots, 0 x_{n}\right\}$ and $0 x_{1}=\sigma(1), \ldots, 0 x_{i-1}=\sigma(i-1) ; 0 x_{i}, \ldots, 0 x_{n-j}$ are orthogonal to $\sigma(i)$, and $0 x_{n-j+1}, \ldots, 0 x_{n}$ form, with $\sigma(i)$, angles less than $\pi / 2-$ $\varepsilon$. It can also be assumed without loss of generality that the origin is the center of $I$. Hence $I$ has the form

$$
I=\left\{x \in \mathbb{R}^{n}:\left|\left(e_{1}, x\right)\right|<\delta_{1}, \ldots,\left|\left(e_{n}, x\right)\right|<\delta_{n}\right\},
$$

where the unit vectors $e_{1}, \ldots, e_{n-k_{E}}$ are parallel to $\sigma(1), \ldots, \sigma\left(n-k_{E}\right)$, respectively, and $\delta_{1}<\cdots<\delta_{n-k_{E}+1}=\cdots=\delta_{n}$.

Let $f: \mathbb{R}^{n} \rightarrow \mathbb{R}$ be a continuous function such that $f(x)=h(x \in I)$, $f(x)=0(x \notin 2 I)$ and $0 \leq f(x) \leq h\left(x \in \mathbb{R}^{n}\right)$.Obviously, $f$ is uniformly continuous too. Since $\mathbb{I}\left(\mathbb{R}^{n}\right)=\mathbb{I}\left(\mathbb{R}^{q}\right) \times \mathbb{I}\left(\mathbb{R}^{n-q}\right)$, by virtue of Proposition 4

$$
M_{\mathbb{I}}\left(h \chi_{I}\right) \leq M_{\mathbb{I}}(f) \leq M_{1}\left[M_{2}(f)\right]
$$

where the operators $M_{1}$ and $M_{2}$ are defined by Proposition 4 .

For $t \in \mathbb{R}^{n-j}$ denote $\Gamma(t)=\left\{y \in \mathbb{R}^{j}:(t, y) \in 2 I\right\}$.

By the condition of the lemma the rectangle $2 I$ is defined by a system of the following type:

$$
\left\{\begin{array}{c}
\left|x_{1}\right|<2 \delta_{1} \\
\ddots \\
\left|x_{i-1}\right|<2 \delta_{i-1} \\
\\
\quad\left|a_{i+1}^{(i)} x_{i}+\cdots+a_{i+1}^{(n-j+1)} x_{n-j+1}+\cdots+a_{i+1}^{(n)} x_{n}\right|<2 \delta_{i+1} \\
\vdots \\
\\
\quad\left|a_{n}^{(i)} x_{i}+\cdots+a_{n}^{(n-j+1)} x_{n-j+1}+\cdots+a_{n}^{(n)} x_{n}\right|<2 \delta_{n},
\end{array}\right.
$$


where $a_{q}^{(p)}$ are the corresponding coordinates of the vectors $e_{i}, \ldots, e_{n}$. Therefore for fixed $t=\left(t_{1}, \ldots, t_{n-j}\right), \Gamma(t)$ is defined by a system of the following type:

$$
\left\{\begin{aligned}
&\left|a_{i}^{(n-j+1)} y_{1}+\cdots+a_{i}^{(n)} y_{j}\right|<2 \delta_{i} \\
&\left|a_{i+1}^{(i)} t_{i}+\cdots+a_{i+1}^{(n-j)} t_{n-j}+a_{i+1}^{(n-j+1)} y_{1}+\cdots+a_{i+1}^{(n)} y_{j}\right|<2 \delta_{i+1} \\
& \vdots \\
&\left|a_{n}^{(i)} t_{i}+\cdots+a_{n}^{(n-j)} t_{n-j}+a_{n}^{(n-j+1)} y_{1}+\cdots+a_{n}^{(n)} y_{j}\right|<2 \delta_{n} .
\end{aligned}\right.
$$

Obviously, $\Gamma(t)$ is a bounded subset of $\mathbb{R}^{j}$ for every $t \in \mathbb{R}^{n-j}$.

Denote by $N$ the set of all vectors $\xi=\left(\xi_{1}, \ldots, \xi_{j}\right) \in \mathbb{R}^{j}$ with the properties: 1$)$ $a_{i}^{(n-j+1)} \xi_{1}+\cdots+a_{i}^{(n)} \xi_{j}=0$, i.e., $\xi$ is orthogonal to the vector $\left(a_{i}^{(n-j+1)}, \ldots, a_{i}^{(n)}\right)$, and 2) $\|\xi\|<\frac{4 n h \delta_{i}}{\sin \varepsilon}$.

For $t \in \mathbb{R}^{n-j}$ with $\Gamma(t) \neq \varnothing$ denote $\Gamma^{*}(t)=\Gamma(t)+N$.

Let $t \in \mathbb{R}^{n-j}, \Gamma(t) \neq \varnothing$. Consider the rotation $\gamma$ in $\mathbb{R}^{j}$ which maps the vector $\left(a_{i}^{(n-j+1)}, \ldots, a_{i}^{(n)}\right)$ into the direction vector of the axis $0 y_{j}-(0, \ldots, 0,1)$. Hence it is easy to see that:

1) $\gamma(N)=\left\{\xi:\|\xi\|<\frac{4 n h \delta_{i}}{\sin \varepsilon}, \xi_{j}=0\right\}$

2) $\gamma(\Gamma(t))$ is defined by a system of the following type:

$$
\left\{\begin{array}{c}
\left|y_{j}\right|<2 \delta_{i} \\
\alpha_{1}<b_{1}^{(1)} y_{1}+\cdots+b_{1}^{(j-1)} y_{j-1}<\alpha_{1}+4 \delta_{i+1} \\
\vdots \\
\alpha_{n-i}<b_{n-i}^{(1)} y_{1}+\cdots+b_{n-i}^{(j-1)} y_{j-1}<\alpha_{n-i}+4 \delta_{n}
\end{array}\right.
$$

Hence, taking into account the equality $\gamma\left(\Gamma^{*}(t)\right)=\gamma(\Gamma(t))+\gamma(N)$, it is easy to see that $\gamma\left(\Gamma^{*}(t)\right)$ is a set of the form $E \times\left(-2 \delta_{i}, 2 \delta_{i}\right)$, where $E$ is an open bounded convex set in $\mathbb{R}^{j}$ containing a ball of radius equal to $4 n h \delta_{i} / \sin \varepsilon$. By the condition of the lemma the axes of $\mathbb{R}^{j} 0 y_{1}, \ldots, 0 y_{j}$ form with the vector $\left(a_{i}^{(n-j+1)}, \ldots, a_{i}^{(n)}\right)$ angles less than $\pi / 2-\varepsilon$. Therefore $\gamma\left(0 y_{1}\right), \ldots, \gamma\left(0 y_{j}\right)$ form with $(0, \ldots, 0,1) \in \mathbb{R}^{j}$ angles less than $\pi / 2-\varepsilon$. Now using Lemma 2 , Proposition 1 and the relations

$$
M_{2}(f)(t, y)=M_{\mathbb{I}\left(\mathbb{R}^{j}\right)}(f(t, \cdot))(y) \leq M_{\mathbb{I}\left(\mathbb{R}^{j}\right)}\left(h \chi_{\Gamma(t)}\right)(y)
$$

for $1 / 2 \leq \lambda<h$ we get

$$
\begin{aligned}
\left|\left\{y \in \mathbb{R}^{j}: M_{2}(f)(t, y)>\lambda\right\}\right|_{j} \leq\left|\left\{M_{\mathbb{I}\left(\mathbb{R}^{j}\right)}\left(h \chi_{\Gamma(t)}\right)>\lambda\right\}\right|_{j} \\
\leq\left|\left\{M_{\mathbb{I}\left(\mathbb{R}^{j}\right)}\left(h \chi_{\Gamma^{*}(t)}\right)>\lambda\right\}\right|_{j}=\left|\left\{M_{\gamma\left(\mathbb{I}\left(\mathbb{R}^{j}\right)\right)}\left(h \chi_{\gamma\left(\Gamma^{*}(t)\right)}\right)>\lambda\right\}\right|_{j} \\
\quad=\left|\left\{M_{\gamma\left(\mathbb{I}\left(\mathbb{R}^{j}\right)\right)}\left(\frac{h}{\lambda} \chi_{\gamma\left(\Gamma^{*}(t)\right)}\right)>1\right\}\right|_{j} \leq d(n) \frac{h}{\lambda}\left|\Gamma^{*}(t)\right|_{j},
\end{aligned}
$$

where $\gamma\left(\mathbb{I}\left(\mathbb{R}^{j}\right)\right)$ denotes the basis for which $\gamma\left(\mathbb{I}\left(\mathbb{R}^{j}\right)\right)(y)=\{\gamma(R): R$ is an $j$ dimensional interval, $x \in \gamma(R)\}\left(y \in \mathbb{R}^{j}\right)$ and $d(n)=n^{2 n} 9^{n}$. 
Since $\sum_{p=i}^{n}\left(a_{q}^{(p)}\right)^{2}=1(i+1 \leq q \leq n)$ and $\delta_{q} \geq \frac{2 n \sqrt{n} h \delta_{i}}{\sin \varepsilon}(i+1 \leq q \leq n)$, we can easily conclude: for $t \in \mathbb{R}^{n-j}$ with $\Gamma(t) \neq \varnothing$

$$
\left\{(t, y): y \in \Gamma^{*}(t)\right\} \subset I^{\prime}
$$

where $I^{\prime}$ is a rectangle defined as

$$
\begin{array}{r}
I^{\prime}=\left\{x \in \mathbb{R}^{n}:\left|\left(e_{1}, x\right)\right|<2 \delta_{1}, \ldots,\left|\left(e_{i}, x\right)\right|<2 \delta_{i},\right. \\
\left.\left|\left(e_{i+1}, x\right)\right|<4 \delta_{i+1}, \ldots,\left|\left(e_{n}, x\right)\right|<4 \delta_{n}\right\} .
\end{array}
$$

Denote $\Gamma^{\prime}(t)=\left\{y \in \mathbb{R}^{j}:(t, y) \in I^{\prime}\right\} \quad\left(t \in \mathbb{R}^{n-j}\right)$. Using (3), we write

$$
\Gamma^{*}(t) \subset \Gamma^{\prime}(t) \text { for } t \in \mathbb{R}^{n-j} \text { with } \Gamma(t) \neq \varnothing .
$$

By virtue of Fubini's theorem, (2) and (3), for $\frac{1}{2} \leq \lambda<h$ we have

$$
\begin{aligned}
\left|\left\{M_{2}(f)>\lambda\right\}\right| & =\int_{\left\{t \in \mathbb{R}^{n-j}: \Gamma(t) \neq \varnothing\right\}}\left|\left\{y \in \mathbb{R}^{j}: M_{2}(f)(t, y)>\lambda\right\}\right|_{j} d t \\
& \leq \int_{\left\{t \in \mathbb{R}^{n-j}: \Gamma(t) \neq \varnothing\right\}} \frac{d(n) h}{\lambda}\left|\Gamma^{*}(t)\right|_{j} d t \\
& \leq \frac{d(n) h}{\lambda} \int_{\left\{t \in \mathbb{R}^{n-j}: \Gamma^{\prime}(t) \neq \varnothing\right\}}\left|\Gamma^{\prime}(t)\right|_{j} d t=d(n) \frac{h}{\lambda}\left|I^{\prime}\right| \\
& =2^{2 n-i} d(n) \frac{h}{\lambda}|I|<d_{1}(n) \frac{h}{\lambda}|I| .
\end{aligned}
$$

By virtue of Proposition $3\left\{M_{2}(f)>1 / 2\right\}$ is bounded. It is also clear that $M_{2}(f)(x) \leq h\left(x \in \mathbb{R}^{n}\right)$. Therefore

$$
\int_{\left\{M_{2}(f)>1 / 2\right\}} M_{2}(f)\left(1+\ln ^{+} 2 M_{2}(f)\right)^{n-j-1}=\alpha<\infty .
$$

By (1), (6) and Proposition 4 we have

$$
\left|\left\{M_{\mathbb{I}}\left(h \chi_{I}\right)>1\right\}\right| \leq\left|\left\{M_{\mathbb{I}}(f)>1\right\}\right| \leq\left|\left\{M_{1}\left[M_{2}(f)\right]>1\right\}\right| \leq c_{3}(n-j) \alpha .
$$

Let $F$ be a distribution function of $M_{2}(f) \chi_{\left\{M_{2}(f)>1 / 2\right\}}$ and $\Phi(\lambda)=\lambda(1+$ $\left.\ln ^{+} 2 \lambda\right)^{n-j-1}$. By (6), Proposition 6 and the obvious equalities $F(\lambda)=F(1 / 2)$ $(0 \leq \lambda \leq 1 / 2), F(\lambda)=0(\lambda>h)$, we can write

$$
\begin{aligned}
\alpha & =\int_{0}^{\infty} F(\lambda) \Phi^{\prime}(\lambda) d \lambda=\int_{0}^{1}+\int_{1}^{h}+\int_{h}^{\infty} \\
& \leq F(1 / 2)+(n-j) \int_{1}^{h} F(\lambda)(1+\ln 2 \lambda)^{n-j-1} d \lambda+0
\end{aligned}
$$




$$
\begin{aligned}
& \leq 2 d_{1}(n) h|I|+(n-j) d_{1}(n) h|I| \int_{1}^{h} \frac{(1+\ln 2 \lambda)^{n-j-1}}{\lambda} d \lambda \\
& \leq 4(n-j) d_{1}(n) h(1+\ln 2 h)^{n-j}|I| .
\end{aligned}
$$

By (7) and (8) we conclude that the lemma is valid in the considered case.

If $\theta \in E_{1, j}(\sigma, \varepsilon)$ where $1 \leq j \leq n-1$, then the proof is analogous to the above-considered case, and if $\theta \in E_{1, n}(\sigma, \varepsilon)$ the assertion follows from Lemma 1. The proof of the lemma is completed.

Lemma 4. Let $I$ be a rectangle in $\mathbb{R}^{n}$ and $h>1$. Then for every $\theta \in \theta\left(\mathbb{R}^{n}\right)$ there is a compact set $S(\theta)$ such that

$$
\left\{M_{\mathbb{I}(\theta)}\left(h \chi_{I}\right) \geq 1\right\} \subset S(\theta), \quad|S(\theta)| \leq 2^{n}\left|\left\{M_{\mathbb{I}(\theta)}\left(h \chi_{I}\right) \geq 1\right\}\right|, \quad S(\theta) \subset 2^{4 n} h I .
$$

Proof. Let $f: \mathbb{R}^{n} \rightarrow \mathbb{R}$ be a continuous function such that $f(x)=h(x \in I)$, $f(x)=0(x \notin 2 I)$ and $0 \leq f(x) \leq h\left(x \in \mathbb{R}^{n}\right)$. Obviously, $f$ is uniformly continuous too.

Assume that $S(\theta)=\left\{M_{\mathbb{I}(\theta)}\left(f \chi_{I}\right) \geq 1\right\}$. By virtue of Proposition $5 M_{\mathbb{I}(\theta)}(f)$ is continuous. Hence taking into account that $S(\theta)=\left\{h \geq M_{\mathbb{I}(\theta)}(f) \geq 1\right\}=$ $M_{\mathbb{I}(\theta)}(f)^{-1}([1, h])$, we conclude that $S(\theta)$ is closed. According to Proposition 3 , $S(\theta)$ is bounded. Hence $S(\theta)$ is compact.

Since $h \chi_{I} \leq f \leq h \chi_{2 I}$, we have

$$
\left\{M_{\mathbb{I}(\theta)}\left(h \chi_{I}\right) \geq 1\right\} \subset S(\theta) \subset\left\{M_{\mathbb{I}(\theta)}\left(h \chi_{2 I}\right) \geq 1\right\} .
$$

By (1) and Proposition 3 we get

$$
\begin{aligned}
S(\theta) \subset\left\{M_{\mathbb{I}(\theta)}\left(h \chi_{2 I}\right) \geq 1\right\} & \subset\left\{M_{\mathbb{I}(\theta)}\left(h \chi_{2 I}\right)>1 / 2\right\} \\
& =\left\{M_{\mathbb{I}(\theta)}\left(2 h \chi_{2 I}\right)>1\right\} \subset\left(2^{n+1} h+1\right) 2 I \subset 2^{4 n} h I .
\end{aligned}
$$

It is easy to verify that $\left\{M_{\mathbb{I}(\theta)}\left(h \chi_{2 I}\right) \geq 1\right\}=H\left[\left\{M_{\mathbb{I}(\theta)}\left(h \chi_{I}\right) \geq 1\right\}\right]$, where $H$ is homothety with center coinciding with the center of $I$ and with coefficient 2. Therefore

$$
\left|\left\{M_{\mathbb{I}(\theta)}\left(h \chi_{2 I}\right) \geq 1\right\}=2^{n}\right|\left\{M_{\mathbb{I}(\theta)}\left(h \chi_{I}\right) \geq 1\right\} \mid .
$$

By (1) and (2) we have

$$
|S(\theta)| \leq 2^{n}\left|\left\{M_{\mathbb{I}(\theta)}\left(h \chi_{I}\right) \geq 1\right\}\right| .
$$

The lemma is proved.

Lemma 5. Let $I$ be a rectangle in $\mathbb{R}^{n}$ with center at the origin and $h>1$. Then for every $E \subset \theta\left(\mathbb{R}^{n}\right), E \neq \varnothing$, there exist $\theta_{1}, \ldots, \theta_{m} \in E$ such that for every $\theta \in E$ there are $i=i_{\theta} \in \overline{1, m}$ and a rotation $\gamma=\gamma_{\theta}$ such that

$$
\left\{M_{\mathbb{I}(\theta)}\left(h \chi_{I}\right) \geq 1\right\} \subset \gamma\left[\left\{M_{\mathbb{I}\left(\theta_{i}\right)}\left(h \chi_{2 I}\right) \geq 1\right\}\right] \text { and } \gamma(2 I) \subset 4 I \text {. }
$$

Proof. As usual by $S O(n)$ we denote the rotation group. For a matrix $A \in$ $S O(n)$ by $\gamma_{A}$ denote the rotation defined by the equality $\gamma_{A}(x)=A x\left(x \in \mathbb{R}^{n}\right)$.

For $A \in S O(n)$, by $\mathbb{I}(A)$ denote the basis for which

$$
\mathbb{I}(A)(x)=\left\{\gamma_{A}(R): R \text { is interval, } x \in \gamma_{A}(R)\right\} \quad\left(x \in \mathbb{R}^{n}\right) .
$$


For the matrix $A \in S O(n)$ denote $\theta_{A}=\left\{\gamma_{A}\left(0 x_{1}\right), \ldots, \gamma_{A}\left(0 x_{n}\right)\right\}$. Let $\widetilde{E}$ be the set of all $A \in S O(n)$ for which $\theta_{A} \in E$.

For $A=\left(a_{i j}\right) \in S O(n)$ and $B=\left(b_{i j}\right) \in S O(n)$ set

$$
\operatorname{dist}(A, B)=\sum_{i=1}^{n} \sum_{j=1}^{n}\left|a_{i j}-b_{i j}\right| .
$$

It is easy to verify that there exists $\varepsilon>0$ such that

$$
I \subset \gamma_{A}(2 I) \subset 4 I \text { if } \operatorname{dist}(A, U)<\varepsilon,
$$

where $U$ denotes the unit matrix.

Let $A, B \in S O(n)$ and $\gamma=\gamma_{B A^{-1}}$. It is easy to check that for any rectangle $J M_{\mathbb{I}(B)}\left(h \chi_{\gamma(J)}\right)(\gamma(x))=M_{\mathbb{I}(A)}\left(h \chi_{J}\right)(x)\left(x \in \mathbb{R}^{n}\right)$ and therefore

$$
\left\{M_{\mathbb{I}(B)}\left(h \chi_{\gamma(J)}\right) \geq 1\right\}=\gamma\left[\left\{M_{\mathbb{I}(A)}\left(h \chi_{J}\right) \geq 1\right\}\right] .
$$

As is easy to see, $\operatorname{dist}(A C, B C) \leq n^{2} \operatorname{dist}(A, B)(A, B, C \in S O(n))$. Therefore $\operatorname{dist}\left(B A^{-1}, U\right) \leq n^{2} \operatorname{dist}(A, B)<\varepsilon$ if $\operatorname{dist}(A, B)<\varepsilon / n^{2}$. Hence, using (2) and (3) we get: if $A, B \in S O(n)$, $\operatorname{dist}(A, B)<\frac{\varepsilon}{n^{2}}$ and $\gamma=\gamma_{B A^{-1}}$, then

$$
\left\{M_{\mathbb{I}(B)}\left(h \chi_{I}\right) \geq 1\right\} \subset\left\{M_{\mathbb{I}(B)}\left(h \chi_{\gamma(2 I)}\right) \geq 1\right\}=\gamma\left[\left\{M_{\mathbb{I}(A)}\left(h \chi_{2 I}\right) \geq 1\right\}\right] .
$$

Since $S O(n)$ is compact with respect to metric (1), there are $A_{1}, \ldots, A_{m} \in E$ which make up a $\varepsilon / n^{2}$-net of the set $\widetilde{E}$. Now, taking into account (2) and (4) and noting that $\mathbb{I}\left(\theta_{A}\right)=\mathbb{I}(A)(A \in S O(n))$, we conclude that $\theta_{1}=\theta_{A_{1}}, \ldots, \theta_{m}=\theta_{A_{m}}$ are the desired frames. The lemma is proved.

We are ready now to begin proving the Lemma on Contrast.

Proof of the Lemma on Contrast. I. Assume without of loss of generality that the origin is the center of $I$.

The following simple fact, which is useful in the construction of various counterexamples, is well known (see, e.g., [2, p. 239]) in the theory of differentiation of integrals : for any interval $J$ in $\mathbb{R}^{n}$ and $\alpha>1$

$$
\left|\left\{M_{\mathbb{I}}\left(\alpha \chi_{J}\right)>1\right\}\right| \geq c_{5}(n) \alpha(\ln \alpha)^{n-1}|J| .
$$

Denote $k=k_{E}$. By Proposition 1 it is sufficient to consider the case where $\Delta_{E}=\left\{0 x_{1}, \ldots, 0 x_{n-k}\right\}$. Clearly, $I$ has the form

$$
I=\gamma_{0}\left[\left(-\delta_{1}, \delta_{1}\right) \times \cdot \times\left(-\delta_{n-k}, \delta_{n-k}\right) \times(-\delta, \delta)^{k}\right],
$$

where $\gamma_{0}$ is the rotation which does not remove the axes $0 x_{1}, \ldots, 0 x_{n-k}$.

Let $\theta \in E$. Obviously, $\theta \supset\left\{0 x_{1}, \ldots, 0 x_{n-k}\right\}$. Consider the rotation $\gamma$ which does not remove $0 x_{1}, \ldots, 0 x_{n-k}$ and maps $0 x_{n-k+1}, \ldots, 0 x_{n}$ into the lines from $\theta \backslash\left\{0 x_{1}, \ldots, 0 x_{n-k}\right\}$. Assume

$$
S(A)=\gamma\left[\left\{M_{\mathbb{I}}\left(h \chi_{Q}\right)>1\right\}\right],
$$

where $Q=\left(-\delta_{1}, \delta_{1}\right) \times \cdots \times\left(-\delta_{n-k}, \delta_{n-k}\right) \times(-\delta / \sqrt{k}, \delta / \sqrt{k})^{k}$. It is easy to see that $\gamma(Q) \subset I$. Hence by Proposition 1 we have

$$
\left\{M_{\mathbb{I}(\theta)}\left(h \chi_{I}\right)>1\right\} \supset\left\{M_{\mathbb{I}(\theta)}\left(h \chi_{\gamma(Q)}\right)>1\right\}=\gamma\left[\left\{M_{\mathbb{I}}\left(h \chi_{Q}\right)>1\right\}\right]=S(\theta) .
$$


By Proposition 3

$$
S(\theta) \subset 2^{2 n} h \gamma(Q) \subset 2^{2 n} h I
$$

and by (1)

$$
|S(\theta)|>c_{5}(n) h(\ln h)^{n-1}|\gamma(Q)|=c_{5}(n) h(\ln h)^{n-1} \frac{|I|}{k^{k / 2}} .
$$

It is obvious that the set $\left\{M_{\mathbb{I}}\left(7 \chi_{Q}>1\right\}\right.$ is open. Thus the sets $S(\theta)(\theta \in E)$ are derived by the rotation of the fixed open set. Therefore, taking into account (4)-(5), it is easy to find the sets $T(\theta)(\theta \in E)$ with properties I.1) and I.2).

II. Due to Lemma 5 there are $\theta_{1}, \ldots, \theta_{m} \in E(\sigma, \varepsilon)$ such that for any given $\theta \in E(\sigma, \varepsilon)$ there exist $i \in \overline{1, m}$ and a rotation $\gamma$ such that

$$
\left\{M_{\mathbb{I}(\theta)}\left(h \chi_{I}\right) \geq 1\right\} \subset \gamma\left[\left\{M_{\mathbb{I}\left(\theta_{i}\right)}\left(h \chi_{2 I}\right) \geq 1\right\}\right] \text { and } \gamma(2 I) \subset 4 I .
$$

According to Lemma 4 , for every $i \in \overline{1, m}$ there is a compact set $S_{i}$ such that

$$
\begin{aligned}
& \left\{M_{\mathbb{I}\left(\theta_{i}\right)}\left(h \chi_{2 I}\right) \geq 1\right\} \subset S_{i}, \\
& \left|S_{i}\right| \leq 2^{n}\left|\left\{M_{\mathbb{I}\left(\theta_{i}\right)}\left(h \chi_{2 I}\right) \geq 1\right\}\right|, \\
& S_{i} \subset 2^{4 n} h(2 I)=2^{4 n+1} h I .
\end{aligned}
$$

Taking into account the inclusion $\left\{M_{\mathbb{I}\left(\theta_{i}\right)}\left(h \chi_{2 I}\right) \geq 1\right\} \subset\left\{M_{\mathbb{I}\left(\theta_{i}\right)}\left(2 h \chi_{2 I}\right)>1\right\}$, using (8) and noting that $r(2 I)=r(I)>\frac{4 n \sqrt{n} h}{\sin \varepsilon}$, from Lemma 3 we have

$$
\left|S_{i}\right| \leq 2^{n} 2^{n} c_{4}(n) h(\ln h)^{n-2}|2 I|=2^{3 n} c_{4}(n) h(\ln h)^{n-2}|I| .
$$

By (6) and (9) we get

$$
\gamma\left(S_{i}\right) \subset \gamma\left(2^{4 n+1} h I\right)=2^{4 n} h \gamma(2 I) \subset 2^{4 n} h(4 I)=2^{4 n+2} h I .
$$

Now taking into account $(6),(7),(10),(11)$ and the properties of compact sets, it is easy to find the sets $T(\theta)(\theta \in E(\sigma, \varepsilon))$ with properties II.1) and II.2). The lemma is proved.

Proof of the Lemma on Distribution. Obviously, there exists an increasing function $f_{*}:(0,1) \rightarrow \mathbb{R}$ such that $\left|\left\{f_{*}>\lambda\right\}\right|_{1}=F(\lambda)$ for any $\lambda \in \mathbb{R}$. Set $E(x)=\left[\left(x_{1}, 1\right) \times(0,1)^{n-1}\right] \cap E$ for $x \in E$. Define the function $f$ as follows: $f(x)=f_{*}(1-|E(x)|)$ when $x \in E,|E(x)|>0$ and $f(x)=0$ otherwise.

Obviously, supp $f \subset E$. It is easy to verify that $f$ is equimeasurable with $f_{*}$ and therefore $F_{f}=F$.

Denote $E_{\alpha}=\left[[\alpha, 1] \times[0,1]^{n-1}\right] \cap E(\alpha \leq 1)$. It is easy to see that for every $\varepsilon>0, f$ is bounded on the set $A_{\varepsilon}=\mathbb{R}^{n} \backslash\left[\alpha_{0}-\varepsilon, \alpha_{0}\right] \times[0,1]^{n-1}$, where $\alpha_{0}=\inf \left\{\alpha \geq 0:\left|E_{\alpha}\right|=0\right\}$. Therefore by virtue of the Jessen-MarcinkiewiczZygmund theorem we easily conclude that $\int f$ is strongly differentiable along every frame. The lemma is proved.

\section{Proof of the Theorem}

First, let us prove the following assertion:

Let $E$ be a nonempty orbit. Then for every $f \in L \backslash L\left(\ln ^{+} L\right)^{n-1}\left(G^{n}\right), f \geq 0$, and $m \in \mathbb{N}$ there are a measurable set $A \in \mathbb{R}^{n}$ and a measurable function $g: \mathbb{R}^{n} \rightarrow \mathbb{R}$ such that: 
1) $A \subset\left\{f \geq 2^{m}\right\}$ and $f \chi_{A}$ is a bounded function;

2) $g$ is equimeasurable with $f \chi_{A}$ and $\operatorname{supp} g \subset G^{n}$;

3) for every $\theta \in E\left|\left\{M_{\mathbb{I}(\theta)}^{(1 / m)}(g)>m\right\}\right|>1-1 / m$;

4) if $E$ is nontrivial, then, in addition, $g$ can be chosen such that, for $\sigma \in$ $\sigma(E)$, an a priori given $g$ satisfies the condition: for every $\theta \in E\left(\sigma, 1 / 2^{m}\right)$ $\left|\left\{M_{\mathbb{I}(\theta)}(g)>1 / 2^{m}\right\}\right|<1 / 2^{m}$.

Proof. Let $E$ be a nontrivial orbit and $\sigma \in \sigma(E)$. Let $r \in \mathbb{N}$. Since $f \in$ $L \backslash L\left(\ln ^{+} L\right)^{n-1}\left(G^{n}\right)$, it is easy to find $p \in \mathbb{N}$, sets $\left\{A_{i}\right\}_{i=1}^{p}$ and numbers $\left\{h_{i}\right\}_{i=1}^{p}$ such that

$$
\begin{aligned}
& \left|A_{i}\right|>0 \quad(i \in \overline{1, p}), \quad A_{i} \cap A_{j}=\varnothing \quad(1 \leq i<j \leq p) \\
& h_{i} \geq 2^{r} \quad(i \in \overline{1, p}) ; \\
& h_{i} \leq f(x)<h_{i}+1 \quad\left(x \in A_{i}, \quad i \in \overline{1, p}\right) \\
& \sum_{i=1}^{p} \frac{h_{i}}{r} \ln ^{n-1} \frac{h_{i}}{r}\left|A_{i}\right|=r \\
& \sum_{i=1}^{p} 2^{r} h_{i} \ln ^{n-2}\left(2^{r} h_{i}\right)\left|A_{i}\right|<\frac{1}{4^{r}}
\end{aligned}
$$

for the rectangle $I_{i}(i \in \overline{1, p})$ with the properties: $I_{i} \in \mathcal{I}(E, \sigma),\left|I_{i}\right|=\left|A_{i}\right|$, $(1 / 2, \ldots, 1 / 2)$ is the center of $I$ and $r(I)=2^{r+3} n \sqrt{n}\left(h_{i}+1\right) / \sin \left(1 / 2^{r}\right)$; the inclusion $2^{7 n+r+1}\left(h_{i}+1\right) I_{i} \subset G^{n}$ is true.

Let us consider numbers $2^{r n}<q_{1}<\cdots<q_{p}$ of the type $2^{n \nu}$ (their choice will be discussed below) and carry out the following construction:

I. for every $i \in \overline{1, p}$, we divide $G^{n}$ into $q_{i}$ equal diadic cubes and denote them by $G_{i, q}\left(q \in \overline{1, q_{i}}\right)$;

II. for $i \in \overline{1, p}$ and $q \in \overline{1, q_{i}}$ assume $I_{i, q}=H_{i, q}\left(I_{i}\right)$, where $H_{i, q}$ is the homothety mapping of $G^{n}$ into $G_{i, q}$;

III. for $i \in \overline{1, p}, q \in \overline{1, q_{i}}$ assume that $\left\{T_{i, q}(\theta): \theta \in E\right\}$ is the collection of sets corresponding to $I=I_{i, q}$ and $h=h_{i} / r$ due to the Lemma on Contrast;

IV. for $i \in \overline{1, p}, q \in \overline{1, q_{i}}$ assume that $\left\{T_{i, q}(\theta): \theta \in E\left(\sigma, 1 / 2^{r}\right)\right\}$ is the collection of sets corresponding to $I=I_{i, q}, h=2^{r+1}\left(h_{i}+1\right)$ and $\varepsilon=1 / 2^{r}$ due to the Lemma on Contrast.

For $\theta \in E \cup E\left(\sigma, 1 / 2^{r}\right)$ denote $\left.T_{i}(\theta)=\bigcup_{q=1}^{q_{i}} T_{i, q}(\theta) i \in \overline{1, p}\right)$. Now if we take into account the structure of sets $T_{i, q}(\theta)$ (see the Lemma on Contrast), then by a rapid increase of numbers $q_{i}$ we can carry out "uniform approximation" of sets $T_{i}(\theta)$ by unions of diadic cubes $G_{i+1, q}$ (from below and from above in the cases $\theta \in E$ and $\theta \in E\left(\sigma, 1 / 2^{r}\right)$, respectively). More exactly, if we denote:

I. for $i \in \overline{1, p-1}$ and $\theta \in E$, by $S_{i}(\theta)$ the union of all $G_{i+1, q}$ that are contained in $T_{i}(\theta)$;

II. for $i \in \overline{1, p-1}$ and $\theta \in E\left(\sigma, 1 / 2^{r}\right)$, by $S_{i}(\theta)$ the union of all $G_{i+1, q}$ that have a nonempty intersection with $T_{i}(\theta)$, 
then, by a rapid increase of numbers $q_{i}$, the following conditions can be fulfilled:

$$
\begin{aligned}
& \left|S_{i}(\theta)\right|>\frac{\left|T_{i}(\theta)\right|}{2} \quad(i \in \overline{1, p-1}, \quad \theta \in E), \\
& \left|S_{i}(\theta)\right|<2\left|T_{i}(\theta)\right| \quad\left(i \in \overline{1, p-1}, \quad \theta \in E\left(\sigma, 1 / 2^{r}\right)\right) .
\end{aligned}
$$

By virtue of the construction and the Lemma on Contrast

$$
\begin{aligned}
& \sum_{q=1}^{q_{i}}\left|I_{i, q}\right|=\left|A_{i}\right| ; \\
& T_{i, q}(\theta) \subset 2^{7 n+r+1}\left(h_{i}+1\right) I_{i, q} \subset G_{i, q} \quad\left(\theta \in E \cup E\left(\sigma, 1 / 2^{r}\right)\right) ; \\
& \left\{M_{\mathbb{I}(\theta)}^{(1 / r)}\left(h_{i} \chi_{I_{i, q}}\right)>r\right\} \supset T_{i, q}(\theta), \\
& \left|T_{i, q}(\theta)\right| \geq c_{1}(n) \frac{h_{i}}{r} \ln ^{n-1} \frac{h_{i}}{r}\left|I_{i, q}\right| ; \quad(\theta \in E) \\
& \left\{M_{\mathbb{I}(\theta)}\left[\left(h_{i}+1\right) \chi_{I_{i, q}}\right]>1 / 2^{r}\right\} \subset T_{i, q}(\theta), \quad\left(\theta \in E\left(\sigma, 1 / 2^{r}\right)\right) \\
& \left|T_{i, q}(\theta)\right| \leq 2^{n} c_{2}(n) 2^{r} h_{i} \ln ^{n-2}\left(2^{r} h_{i}\right)\left|I_{i, q}\right| .
\end{aligned}
$$

Denote

$$
J_{i}=\bigcup_{q=1}^{q_{i}} I_{i, q} \backslash \bigcup_{j=i+1}^{p} \bigcup_{q=1}^{q_{j}} I_{j, q}(i \in \overline{1, p-1}), \quad J_{p}=\bigcup_{q=1}^{q_{p}} I_{p, q} .
$$

Let $A_{i}^{\prime}(i \in \overline{1, p})$ be sets such that $A_{i}^{\prime} \subset A_{i},\left|A_{i}^{\prime}\right|=\left|J_{i}\right|$ and $A=\bigcup_{i=1}^{r} A_{i}^{\prime}$. Obviously, $A \subset\left\{f \geq 2^{r}\right\}$ and $f \chi_{A}$ is bounded.

Due to the Lemma on Distribution there exists an equimeasurable with $f \chi_{A_{i}^{\prime}}$ $(i \in \overline{1, p})$ function $v_{i}$ with $\operatorname{supp} v_{i}=J_{i}$. Set $v=\sum_{i=1}^{r} v_{i}$. It is easy to see that $v$ is equimeasurable with $f \chi_{A}$ and

$$
\sup _{1 \leq i \leq p} \sum_{q=1}^{q_{i}} h_{i} \chi_{I_{i, q}} \leq v \leq \sup _{1 \leq i \leq p} \sum_{q=1}^{q_{i}}\left(h_{i}+1\right) \chi_{I_{i, q}}
$$

Let $\theta \in E$. Assume $S_{p}(\theta)=T_{p}(\theta)$. It is not difficult to see that the sets $G^{n} \backslash S_{i}(\theta)$ are probabilistically independent. Therefore, using (4), we have

$$
\left|\bigcap_{i=1}^{p}\left[G^{n} \backslash S_{i}(\theta)\right]\right|=\prod_{i=1}^{p}\left|G^{n} \backslash S_{i}(\theta)\right|<\prod_{i=1}^{p}\left(1-\frac{\left|T_{i}(\theta)\right|}{2}\right) .
$$

By virtue of (6)-(8) for $i \in \overline{1, p-1}$

$$
\left|T_{i}(\theta)\right|=\sum_{q=1}^{q_{i}}\left|T_{i, q}(\theta)\right| \geq c_{1}(n) \frac{h_{i}}{r} \ln ^{n-1} \frac{h_{i}}{r}\left|A_{i}\right|,
$$

therefore from $(2),(10)$ and the inequality $\ln (1-\alpha)<-\alpha(0<\alpha<1)$ we have

$$
\left|\bigcap_{i=1}^{p}\left[G^{n} \backslash S_{i}(\theta)\right]\right|<\frac{1}{2^{c_{6}(n) r}} .
$$


By virtue of the construction

$$
\left\{M_{\mathbb{I}(\theta)}^{(1 / r)}(v)>r\right\} \supset \bigcup_{i=1}^{p}\left\{M_{\mathbb{I}(\theta)}^{(1 / r)}\left(\sum_{q=1}^{q_{i}} h_{i} \chi_{I_{i, q}}\right)>r\right\} \supset \bigcup_{i=1}^{p} T_{i}(\theta) \supset \bigcup_{i=1}^{p} S_{i}(\theta) .
$$

Thus

$$
\left|\left\{M_{\mathbb{I}(\theta)}^{(1 / r)}(v)>r\right\}\right|>1-\frac{1}{2^{c_{6}(n) r}} .
$$

Let $\theta \in E\left(\sigma, 1 / 2^{r}\right)$. Denote

$$
N_{1}=\overline{1, q_{1}}, \quad t_{1}=\sum_{q=1}^{q_{1}}\left(h_{i}+1\right) \chi_{I_{1, q}}
$$

and for $i \in \overline{2, p}$

$$
\begin{aligned}
& N_{i}=\left\{q \in \overline{1, q_{i}}: G_{i, q} \cap \bigcup_{j=1}^{i-1} T_{j}(\theta)=\varnothing\right\}, \\
& N_{i}^{*}=\left\{q \in \overline{1, q_{i}}: G_{i, q} \cap \bigcup_{j=1}^{i-1} T_{j}(\theta) \neq \varnothing\right\}, \\
& t_{i}=\sum_{q \in N_{i}}\left(h_{i}+1\right) \chi_{I_{i, q}}, \quad t_{i}^{*}=\sum_{q \in N_{i}^{*}}\left(h_{i}+1\right) \chi_{I_{i, q}} .
\end{aligned}
$$

Let $t=\sum_{i=1}^{p} t_{i}$ and $t^{*}=\sup _{2 \leq i \leq p} t_{i}^{*}$. Obviously, $v \leq t+t^{*}$. Clearly, the functions $\left(h_{i}+\right.$ 1) $\chi_{I_{i, q}}$ belong to $\bar{L}\left(\mathbb{R}^{n}\right)$. Therefore from $(7)$, (9) and the Lemma on Separation we obtain

$$
\left\{M_{\mathbb{I}(\theta)}\left(t_{i}\right)>1 / 2^{r+1}\right\} \subset \bigcup_{q \in N_{i}} T_{i, q}(\theta) \quad(i \in \overline{1, p}) .
$$

We easily observe that

$$
\left[\bigcup_{q \in N_{i}} T_{i, q}(\theta)\right] \cap\left[\bigcup_{q \in N_{j}} T_{j, q}(\theta)\right]=\varnothing \quad(i \neq j)
$$

Thus again by virtue of the Lemma on Separation

$$
\left\{M_{\mathbb{I}(\theta)}(t)>1 / 2^{r+1}\right\}=\bigcup_{i=1}^{p}\left\{M_{\mathbb{I}(\theta)}\left(t_{i}\right)>1 / 2^{r+1}\right\} .
$$

(13), (12), (6), (9), and (3) imply

$$
\begin{aligned}
\mid\left\{M_{\mathbb{I}(\theta)}(t)\right. & \left.>1 / 2^{r+1}\right\}\left|\leq \sum_{i=1}^{p} \sum_{q \in N_{i}}\right| T_{i, q}(\theta) \mid \\
\leq & \sum_{i=1}^{p} \sum_{q=1}^{q_{i}}\left|T_{i, q}(\theta)\right| \leq \sum_{i=1}^{p} 2^{n} c_{2}(n) 2^{r} h_{i} \ln ^{n-2}\left(2^{r} h_{i}\right)\left|A_{i}\right|<\frac{2^{n} c_{2}(n)}{4^{r}} .
\end{aligned}
$$


Let $i \in \overline{2, p}$. If $j>i$, then every $G_{i, q}$ is included in some $G_{j, q}$. Therefore $\bigcup_{q \in N_{i}^{*}} G_{i, q} \subset \bigcup_{j=1}^{i-1} S_{j}(\theta)$, from which, using (5), we have $\left|\bigcup_{q \in N_{i}^{*}} G_{i, q}\right| \leq \sum_{j=1}^{i-1} 2\left|T_{j}(\theta)\right|$. This with (9), (6) and (3) gives

$$
\begin{gathered}
\left|\operatorname{supp} t_{i}^{*}\right|=\left|\bigcup_{q \in N_{i}^{*}} I_{i, q}\right|=\left|\bigcup_{q=1}^{q_{i}} I_{i, q}\right|\left|\bigcup_{q \in N_{i}^{*}} G_{i, q}\right| \leq\left|A_{i}\right| \sum_{j=1}^{i-1} 2\left|T_{j}(\theta)\right| \\
\leq 2\left|A_{i}\right| \sum_{j=1}^{p} 2^{n} c_{2}(n) 2^{r} h_{j} \ln ^{n-2}\left(2^{r} h_{j}\right)\left|A_{j}\right| \leq \frac{2^{n+1} c_{2}(n)}{4^{r}}\left|A_{i}\right| .
\end{gathered}
$$

By virtue of the strong maximal inequality (see e.g. [3, p. 51]), (15), (1), and (2) we have

$$
\begin{aligned}
&\left|\left\{M_{\mathbb{I}(\theta)}\left(t^{*}\right)>1 / 2^{r+1}\right\}\right| \leq 2^{n} c_{3}(n) \int_{\mathbb{R}^{n}} 2^{r+1} t^{*} \ln ^{n-1}\left(2^{r+2} t^{*}\right) \\
& \leq 2^{n} c_{3}(n) \sum_{i=2}^{p} 2^{r+1}\left(h_{i}+1\right) \ln ^{n-1}\left[2^{r+1}\left(h_{i}+1\right)\right]\left|\operatorname{supp} t_{i}^{*}\right| \\
& \leq c_{7}(n) \sum_{i=1}^{p} 2^{r} h_{i} \ln ^{n-1}\left(2^{r} h_{i}\right) \frac{\left|A_{i}\right|}{4^{r}} \\
& \leq \frac{c_{8}(n) r}{2^{r}} \sum_{i=1}^{p} \frac{h_{i}}{r} \ln ^{n-1} \frac{h_{i}}{r}\left|A_{i}\right|=\frac{c_{8}(n) r^{2}}{2^{r}} .
\end{aligned}
$$

Taking into account (14), (16) and the inequality $v \leq t+t^{*}$, we write

$$
\left|\left\{M_{\mathbb{I}(\theta)}(v)>1 / 2^{r}\right\}\right| \leq \frac{2^{n+1} c_{2}(n)}{4^{r}}+\frac{c_{8}(n) r^{2}}{2^{r}} .
$$

As $r \in \mathbb{N}$ is arbitrary, by (11) and (17) we conclude that in the case of a nontrivial orbit the assertion is proved.

If from the above arguments we remove those connected with "good" frames and assume that $E=\theta\left(\mathbb{R}^{n}\right)$, then we can prove the remaining case: $E=\theta\left(\mathbb{R}^{n}\right)$. The assertion is proved.

Now let us proceed to a direct proof of the theorem.

First, let us consider the case $E=\bigcup_{i=0}^{p} E_{i}$, where $E_{i}(i \in \overline{0, p})$ are nontrivial orbits. Assume $\sigma_{i} \in \sigma(E)(i \in \overline{0, p})$ and let $f \in L \backslash\left(\ln ^{+} L\right)^{n-1}\left(G^{n}\right)$.

Denote $m^{*}=m(\bmod p+1)(m \in \mathbb{N})$. By virtue of the assertion proved above there are a sequence of sets $A_{m} \subset G^{n}$ and a sequence of functions $g_{m} \in L\left(G^{n}\right)$ such that

$$
\begin{aligned}
& f \chi_{A_{m}} \text { is bounded } \quad(m \in \mathbb{N}), \\
& A_{m} \cap A_{m^{\prime}}=\varnothing \quad\left(m \neq m^{\prime}\right), \\
& g_{m} \text { is equimeasurable with } f \chi_{A_{m}} \quad(m \in \mathbb{N}),
\end{aligned}
$$




$$
\begin{aligned}
& \left|\left\{M_{\mathbb{I}(\theta)}^{(1 / m)}\left(g_{m}\right)>m\right\}\right|>1-\frac{1}{m} \quad\left(m \in \mathbb{N}, \quad \theta \in E_{m^{*}}\right), \\
& \left|\left\{M_{\mathbb{I}(\theta)}\left(g_{m}\right)>1 / 2^{m}\right\}\right|<\frac{1}{2^{m}} \quad\left(m \in \mathbb{N}, \quad \theta \in E_{m^{*}}\left(\sigma_{m^{*}}, 1 / 2^{m}\right)\right) .
\end{aligned}
$$

Set $g^{*}=\sup _{m \in \mathbb{N}} g_{m}$. Let $\theta \in E$. Then from (4) we have

$$
\left|\varlimsup_{m \rightarrow \infty}\left\{M_{\mathbb{I}(\theta)}^{(1 / m)}\left(g_{m}\right)>m\right\}\right|=1 .
$$

Therefore

$$
\bar{D}_{\mathbb{I}(\theta)}\left(\int g^{*}, x\right)=\infty \quad \text { a.e. on } G^{n} .
$$

Let now $\theta \notin E$. It is clear that

$$
\theta\left(\mathbb{R}^{n}\right) \backslash E=\bigcap_{i=0}^{p} \bigcup_{m(\bmod p+1)=i} E_{m^{*}}\left(\sigma_{m^{*}}, 1 / 2^{m}\right)
$$

and therefore $\theta \in E_{m^{*}}\left(\sigma_{m^{*}}, 1 / 2^{m}\right)$ for $m$ large enough. Consequently, there is (see $(5)) m(\theta) \in \mathbb{N}$ such that $\left|\left\{M_{\mathbb{I}(\theta)}\left(g_{m}\right)>1 / 2^{m}\right\}\right|<\frac{1}{2^{m}}$ when $m>m(\theta)$. Hence $\left|\varlimsup_{m \rightarrow \infty}\left\{M_{\mathbb{I}(\theta)}\left(g_{m}\right)>1 / 2^{m}\right\}\right|=0$. ¿From this, (1) and (3) we obtain

$$
M_{\mathbb{I}(\theta)}\left(g^{*}\right)(x) \leq \sum_{m=1}^{\infty} M_{\mathbb{I}(\theta)}\left(g_{m}\right)(x)<\infty \text { a.e. on } G^{n} .
$$

Therefore

$$
0 \leq \underline{D}_{\mathbb{I}(\theta)}\left(\int g^{*}, x\right) \leq \bar{D}_{\mathbb{I}(\theta)}\left(\int g^{*}, x\right) \leq M_{\mathbb{I}(\theta)}\left(g^{*}\right)(x)<\infty \text { a.e. on } G^{n} .
$$

Now according to Guzmán-Menargues' theorem (see [3, p. 106]) we conclude

$$
\int g^{*} \text { is strongly differentiable along } \theta \text {. }
$$

Taking into account the decomposition $g^{*}=\sum_{m=1}^{\infty} g_{m} \chi_{Q_{m}}$, where $Q_{m}=\{x$ : $\left.\sup _{1 \leq i \leq m-1} g_{i}(x)<g^{*}(x), g_{m}(x)=g^{*}(x)\right\}$, and using (2) and (3), it is not difficult to see that $\left|\left\{\lambda_{1}<g^{*} \leq \lambda_{2}\right\}\right| \leq\left|\left\{\lambda_{1}<f \leq \lambda_{2}\right\}\right|\left(\lambda_{1} \leq \lambda_{2}\right)$. Therefore the function

$$
F(\lambda)= \begin{cases}1, & \lambda<0 \\ F_{f}(\lambda)-F_{g^{*}}(\lambda), & \lambda \geq 0\end{cases}
$$

is a distribution. Furthermore, according to the Lemma on Distribution there is a function $g_{*}$ such that $F_{g_{*}}=F, \operatorname{supp} g_{*} \subset G^{n} \backslash \operatorname{supp} g^{*}$ and $g_{*}$ for every $\theta \in \theta\left(\mathbb{R}^{n}\right)$

$$
\int g_{*} \text { is strongly differentiable along } \theta \text {. }
$$

Set $g=g^{*}+g_{*}$. Obviously, $g$ is equimeasurable with $f$. Hence (6), (7), and (9) imply that $g$ is the desired function. Thus the theorem is proved in the considered case. 
If from the above arguments we remove those connected with differentiability and assume that $E=E_{0}=\theta\left(\mathbb{R}^{n}\right)$, then we can prove the theorem in the case $E=\theta\left(\mathbb{R}^{n}\right)$.

In the remaining case $E=\varnothing$, it is sufficient to use the Lemma on Distribution. The theorem is proved.

\section{REFERENCES}

1. B. Jessen, J. Marcinkiewicz, and A. Zygmund, Note on the differentiability of multiple integrals. Fund. Math. 25(1935), 217-234.

2. S. SAKS, On the strong derivatives of functions of intervals, Fund. Math. 25(1935), 235252.

3. M. DE Guzmán, Differentiation of integrals in $\mathbb{R}^{n}$. Lecture Notes in Math. 481, Springer, 1975.

4. J. M. Marstrand, A counter-example in the theory of strong differentiability. Bull. London Math. Soc. 9(1977), 209-211.

5. B. Lopez-Melero, A negative result in differentiation theory. Studia Math. 72(1982), No. 2, 173-182.

6. G. L. LePsveridze, On strong differentiation of integrals along different directions. Georgian Math. J. 2(1995), No. 6, 613-631.

7. G. G. Oniani, On the differentiability of integrals with respect to the bases $B_{2}(\theta)$. East J. Approx. 3(1997), No. 3, 275-301 (Erratum in East J. Approx. 7(2001), No. 3, 371-373).

8. A. S. Stokolos, On a problem of A. Zygmund. (Russian) Mat. Zametki 64(1998), No. 5, 749-762; English transl.: Math. Notes 64(1998), No. 5-6, 646-657 (1999)

9. G. G. Oniani, On R-sets, Bull. Georgian Acad. Sci. 157(1998), No. 2, 181-182.

10. G. G. Oniani, On the strong differentiation of multiple integrals along different frames. Bull. Georgian Acad. Sci. 161(2000), No. 3, 380-381.

11. G. L. LePsveridze, A note on the strong differentiability of integrals along different directions in $\mathbb{R}^{n}(n \geq 3)$. Proc. A. Razmadze Math. Inst. 121(1999) 155-156.

12. A. S. Stokolos, Differentiation of integrals of uniformly measurable functions. (Russian) Mat. Zametki 37(1985), No. 5, 667-675; English transl.: Math. Notes 37(1985), 364-368.

13. G. G. Oniani, Differentiation of Lebesgue integrals. (Russian) Tbilisi University Press, Tbilisi, 1998.

14. G. G. Oniani, On the differentiability of integrals along different frames. Bull. Georgian Acad. Sci. 156(1998), No. 3, 366-368.

15. G. G. OnIANi, On the integrability of strong maximal functions corresponding to different frames. Georgian Math. J. 6(1999), No. 2, 149-169.

(Received 15.09.2004)

Authors' address:

Department of Physics and Mathematics

Kutaisi State University

59, Tamar Mepe St., Kutaisi 4600

Georgia

E-mail: tava73@mail.ru 